\title{
Scaling relations in early-type galaxies from integral-field stellar kinematics
}

M. Cappellari, ${ }^{1}$ N. Scott,${ }^{1}$ K. Alatalo, ${ }^{2}$ L. Blitz,${ }^{2}$ M. Bois, ${ }^{3}$ F. Bournaud, ${ }^{4}$ M. Bureau, ${ }^{1}$ R. L. Davies, ${ }^{1}$ T. A. Davis ${ }^{1}$ P. T. de Zeeuw, ${ }^{5,14}$ E. Emsellem, ${ }^{5,3}$ J. Falcon-Barroso, ${ }^{6}$ S. Khochfar, ${ }^{7}$ D. Krajnovic, ${ }^{1}$ H. Kuntschner, ${ }^{5}$ P.-Y. Lablanche,${ }^{3}$ R. M. McDermid, ${ }^{8}$ R. Morganti, ${ }^{9}$ T. Naab, ${ }^{10}$ M. Sarzi, ${ }^{11}$ P. Serra, ${ }^{9}$ R. C. E. van den Bosch, $^{12}$ G. van de Ven, ${ }^{13}$ A. Weijmans ${ }^{14}$ and L. M. Young ${ }^{15}$

${ }^{1}$ University of Oxford, UK; ${ }^{2}$ University of California, Berkeley, USA $;{ }^{3}$ Université de Lyon, France; ${ }^{4}$ Université Paris Diderot, France; ${ }^{5}$ ESO, Garching, Germany; ${ }^{6}$ IAC, La Laguna, Spain; ${ }^{7}$ MPI for Extraterrestrial Physics, Garching, Germany; ${ }^{8}$ Gemini Observatory, Hilo, USA; ${ }^{9}$ ASTRON, Dwingeloo, The Netherlands; ${ }^{10}$ Universitäts-Sternwarte München, Germany; ${ }^{11}$ University of Hertfordshire, Hatfield, UK; ${ }^{12}$ The University of Texas, Austin, USA; ${ }^{13}$ IAS,

Princeton, USA; ${ }^{14}$ Leiden University, The Netherlands; ${ }^{15}$ New Mexico Tech, Socorro, USA

Early-type galaxies (ETGs) satisfy a now classic scaling relation $R_{\mathrm{e}} \propto \sigma_{\mathrm{e}}^{1.2} I_{\mathrm{e}}^{-0.8}$, the Fundamental Plane (FP; Djorgovski \& Davis 1987; Dressler et al. 1987), between their size, stellar velocity dispersion and mean surface brightness. A significant effort has been devoted in the past twenty years to try to understand why the coefficients of the relation are not the ones predicted by the virial theorem $R_{\mathrm{e}} \propto \sigma_{\mathrm{e}}^{2} I_{\mathrm{e}}^{-1}$.

Recent studies, using independent approaches from either (i) detailed dynamical models or (ii) strong galaxy lensing, point to a genuine variation of the mass-to-light ratio $M / L$ in galaxies as the reason for nearly all the observed 'tilt' in the FP (e.g. Cappellari et al. 2006; Bolton et al. 2008). However these studies are limited by a small and biased sample or are restricted to only the most massive ETGs respectively.

We overcome both limitations by modeling the stellar dynamics, using axisymmetric Jeans anisotropic models (JAM; Cappellari 2008), for the $K$-band selected, volumelimited $\mathrm{ATLAS}^{3 D}$ sample of 263 nearby ETGs, spanning a large range of masses and with $60<\sigma_{\mathrm{e}}<350 \mathrm{~km} \mathrm{~s}^{-1}$. A key for the project is the availability for all galaxies of high-quality integral-field kinematics observed with the SAURON spectrograph and detailed Multi-Gaussian Expansion (Emsellem et al. 1994) models of the photometry.

We confirm the genuine $M / L$ variation and construct both the FP and the More FP (MFP; Bolton et al. 2007) for the ATLAS ${ }^{3 D}$ sample, relating the mean surface density $\Sigma_{\mathrm{e}} \equiv I_{\mathrm{e}} \times(M / L)_{\mathrm{JAM}}, \sigma_{\mathrm{e}}$ and $R_{\mathrm{e}}$. Our MFP produces a relation as tight as the FP over the full mass range. We compare the global $(M / L)_{\text {JAM }}$ variation among galaxies with predictions from two-SSP stellar population models and find that variations of both dark matter (or IMF) and population are required to explain the observations.

\section{References}

Bolton, A. S., et al. 2008, ApJ, 684, 248

Bolton, A. S., Burles, S., Treu, T., Koopmans, L. V. E., \& Moustakas, L. A. 2007, ApJ, 665, L105

Cappellari, M., et al. 2006, MNRAS, 366, 1126

Cappellari, M. 2008, MNRAS, 390, 71

Djorgovski, S. \& Davis, M. 1987, ApJ, 313, 59

Dressler, A., et al. 1987, ApJ, 313, 42

Emsellem, E., Monnet, G., \& Bacon, R. 1994, A\& A, 285, 723 